\title{
RIGID BODY STABILIZATION UNDER TIME-VARYING PERTURBATIONS WITH ZERO MEAN VALUES
}

\author{
Alexander Aleksandrov \\ ITMO University \\ Russia \\ a.u.aleksandrov@spbu.ru
}

Article history:

Received 19.11.2017, Accepted: 15.05.2018

\begin{abstract}
The problem of monoaxial attitude control of a rigid body subjected to nonstationary perturbations is investigated. The control torque consists of a dissipative component and a restoring one. The cases of linear and nonlinear restoring and perturbing torques are analyzed. Two theorems on asymptotic stability of the programmed orientation are proved. The results of a numerical simulation are presented to confirm the conclusions obtained analytically.
\end{abstract}

\section{Key words}

Rigid body, monoaxial stabilization, time-varying perturbations, Lyapunov function, asymptotic stability, averaging method.

\section{Introduction}

Success in the study of physical and mechanical problems essentially depends on the quality of the constructed mathematical model. When constructing a mathematical model describing the dynamics of a mechanical system, we inevitably confine ourselves to take into account main acting forces and torques, relating all the others to the category of disturbing ones.

In some cases, the structure of perturbing forces and torques is known, and their influence can be taken into account using perturbation techniques [Bogoliubov and Mitropolsky, 1961; Nayfeh, 1981; Markeyev, 2011]. Methods of perturbation theory are potent for studying a variety of dynamic problems. In particular, they are widely and successfully used in the problems of the dynamics of artificial and natural celestial bodies [Akulenko, Leshchenko and Chernous'ko, 1986; Tikhonov, 2002; Tikhonov, 2004; Krasilnikov and Amelin, 2016].

\author{
Alexey Tikhonov \\ Saint Petersburg State University \\ Saint Petersburg Mining University \\ Russia \\ a.tikhonov@spbu.ru
}

In this case, the greatest difficulty may occur due to time-varying perturbations.

A powerful tool for the stability analysis of nonstationary systems is the averaging method, see [Bogoliubov and Mitropolsky, 1961; Guckenheimer and Holmes, 1983; Grebennikov, 1986; Khapaev, 1993; Manevich, Smirnov and Romeo, 2016]. This method permits us to reduce investigation of stability of timevarying systems to that of corresponding time-invariant averaged systems, possibly resulting in an essential simplification.

However, it should be noted that the application of the averaging technique is well developed only for the case where considered systems are fast time-varying. In this case the averaging technique is widely used in a variety of dynamics and control problems [Tikhonov, 2005; Ovchinnikov, Roldugin and Penkov, 2012; Kosjakov, E.A. and Tikhonov, 2015; Kovaleva, 2016; Chikhachev, 2017].

In [Aleksandrov, 1996; Aleksandrov, 2001], an original approach to Lyapunov functions constructing was proposed, and, on the basis of the approach, new stability conditions for nonlinear time-varying systems were found. The results of [Aleksandrov, 1996; Aleksandrov, 2001] were further developed in [Peuteman and Aeyels, 1999; Peuteman and Aeyels, 2011; Tikhomirov, 2007; Aleksandrov, Aleksandrova and Zhabko, 2013].

Compared with known stability conditions obtained with the aid of the averaging method, the principal novelty of the results of [Aleksandrov, 1996; Aleksandrov, 2001; Peuteman and Aeyels, 1999; Peuteman and Aeyels, 2011; Tikhomirov, 2007; Aleksandrov, Aleksandrova and Zhabko, 2013] is that, to guarantee the asymptotic stability for considered systems, right-hand sides of the systems need not be fast time-varying.

In the present contribution, the attitude dynamics and control of a rigid body under the influence of a non- 
stationary perturbing torque are investigated. No limitations are imposed on the rate of time-varying in the perturbing torque. The problem of monoaxial stabilization of a rigid body is studied. The control torque consists of a dissipative component and a restoring one. The cases of linear and nonlinear restoring and perturbing torques are analyzed. Two theorems on asymptotic stability of the programmed orientation are proved.

\section{Statement of the Problem}

Consider a rigid body rotating around its mass center $O$ with angular velocity $\boldsymbol{\omega}$. Let the axes $O x y z$ be principal central axes of inertia of the body. Differential equations describing the attitude motion of the body under control torque $\mathbf{M}$ have the form

$$
\Theta \dot{\omega}+\omega \times \Theta \omega=M
$$

where $\boldsymbol{\Theta}=\operatorname{diag}\{A, B, C\}$ is a body inertia tensor in the axes $O x y z$, see [Schaub and Junkins, 2009].

Let unit vectors $\mathbf{s}$ and $\mathbf{r}$ be given, and vector $\mathbf{s}$ be constant in the inertial space and vector $\mathbf{r}$ be constant in the body-fixed frame. Then vector s rotates with respect to coordinate system $O x y z$ with angular velocity $-\boldsymbol{\omega}$. Hence,

$$
\dot{\mathbf{s}}=-\boldsymbol{\omega} \times \mathbf{s} .
$$

Thus, we will study the system consisting of the Euler dynamic equations (1) and the Poisson kinematic equations (2).

Consider the problem of monoaxial stabilization of the body [Zubov, 1978]: it is required to design a control torque $M$ ensuring that system (1), (2) admits the asymptotically stable equilibrium position

$$
\boldsymbol{\omega}=\mathbf{0}, \quad \mathbf{s}=\mathbf{r} .
$$

It is known, see [Zubov, 1978], that the torque $\mathbf{M}$ can be chosen as the sum of dissipative component $\mathbf{M}_{d}$ and restoring one $\mathbf{M}_{r}: \mathbf{M}=\mathbf{M}_{d}+\mathbf{M}_{r}$, where

$$
\mathbf{M}_{d}=h \mathbf{D} \boldsymbol{\omega}, \quad \mathbf{M}_{r}=-a\|\mathbf{s}-\mathbf{r}\|^{\mu-1} \mathbf{s} \times \mathbf{r},
$$

$h>0, \mu \geq 1, a>0, \mathbf{D}$ is a constant symmetric negative definite matrix, and $\|\cdot\|$ denotes the Euclidean norm of a vector.

In the present paper, we consider the case where, along with the control torque $\mathbf{M}$, a perturbing torque $\widetilde{\mathrm{M}}$ acts on the body. Let

$$
\widetilde{\mathbf{M}}=\boldsymbol{\Phi}(t) \mathbf{Q}(\mathbf{s}-\mathbf{r}) .
$$

Here $\boldsymbol{\Phi}(t) \in \mathbb{R}^{3 \times k}$ is a continuous and bounded for $t \geq 0$ matrix, whereas components of the vector $\mathbf{Q}(\mathbf{u}) \in \mathbb{R}^{k}$ are continuously differentiable for $\mathbf{u} \in \mathbb{R}^{3}$ homogeneous of the order $\mu$ functions.

Thus, system (1) can be rewritten as follows

$$
\begin{aligned}
& \boldsymbol{\Theta} \dot{\boldsymbol{\omega}}+\boldsymbol{\omega} \times \boldsymbol{\Theta} \boldsymbol{\omega}=h \mathbf{D} \boldsymbol{\omega} \\
& -a\|\mathbf{s}-\mathbf{r}\|^{\mu-1} \mathbf{s} \times \mathbf{r}+\boldsymbol{\Phi}(t) \mathbf{Q}(\mathbf{s}-\mathbf{r}) .
\end{aligned}
$$

Assumption 1. Let

$$
\frac{1}{T} \int_{t}^{t+T} \boldsymbol{\Phi}(\tau) d \tau \rightarrow 0 \text { as } \quad T \rightarrow+\infty
$$

uniformly with respect to $t \geq 0$.

Assumption 1 is a classical condition of the averaging method. It is well known that the condition is fulfilled for periodic, quasiperiodic and almost periodic functions with zero mean values, see [Bogoliubov and Mitropolsky, 1961]. Moreover, it is fulfilled for some ergodic classes of recurrent functions [Zubov, 1999].

Functions satisfying Assumption 1 are widely used in problems of celestial mechanics [Grebennikov, 1986; Khapaev, 1993], electrodynamics [Khapaev, 1993; Mayu-shan and Altsybeyev, 2016], radio engineering [Bogoliubov and Mitropolsky, 1961], population dynamics [Britton, 2003], etc.

It is worth noting that torques of the form (4) are often occur in mathematical models of satellites moving in circular or elliptic orbits, see [Antipov and Tikhonov, 2014; Giri and Sinha, 2017].

In this contribution, we will look for conditions under which perturbations do not disturb asymptotic stability of the equilibrium position (3).

\section{Linear Restoring and Perturbing Torques}

Consider the case where $\mu=1$, and restoring and perturbing torques are linear. Then system (5) takes the form

$$
\boldsymbol{\Theta} \dot{\boldsymbol{\omega}}+\boldsymbol{\omega} \times \boldsymbol{\Theta} \boldsymbol{\omega}=h \mathbf{D} \boldsymbol{\omega}-a \mathbf{s} \times \mathbf{r}+\boldsymbol{\Phi}(t)(\mathbf{s}-\mathbf{r}) .
$$

Thus, here $\boldsymbol{\Phi}(t) \in \mathbb{R}^{3 \times 3}$.

Theorem 1. Let Assumption 1 be fulfilled. Then there exists a number $h_{0}>0$ such that the equilibrium position (3) of system (2), (6) is asymptotically stable for any $h \geq h_{0}$.

Proof. Construct a Lyapunov function candidate for system (2), (6) on the basis of a synthesis of the approaches developed in [Antonchik, 1983; Kosov, 2007; 
Aleksandrov, Kosov and Chen, 2011] and in [Aleksandrov, 1996; Tikhomirov, 2007].

First, using the results of [Antonchik, 1983; Kosov, 2007; Aleksandrov, Kosov and Chen, 2011], consider the function

$$
\begin{aligned}
V_{1}(\boldsymbol{\omega}, \mathbf{s})= & \frac{\gamma}{2} \boldsymbol{\omega}^{\top} \boldsymbol{\Theta} \boldsymbol{\omega}+\frac{1}{2}\|\mathbf{s}-\mathbf{r}\|^{2} \\
& -\frac{1}{h}(\mathbf{s} \times \mathbf{r})^{\top} \mathbf{D}^{-1} \boldsymbol{\Theta} \boldsymbol{\omega}
\end{aligned}
$$

where $\gamma$ is a positive parameter.

Differentiating $V_{1}(\boldsymbol{\omega}, \mathbf{s})$ with respect to system (2), (6), we obtain

$$
\begin{aligned}
& \dot{V}_{1}=\gamma h \boldsymbol{\omega}^{\top} \mathbf{D} \boldsymbol{\omega}-\gamma a \boldsymbol{\omega}^{\top}(\mathbf{s} \times \mathbf{r}) \\
& +\gamma \boldsymbol{\omega}^{\top} \mathbf{\Phi}(t)(\mathbf{s}-\mathbf{r})+\frac{1}{h}((\boldsymbol{\omega} \times \mathbf{s}) \times \mathbf{r})^{\top} \mathbf{D}^{-1} \boldsymbol{\Theta} \boldsymbol{\omega} \\
& +\frac{1}{h}(\mathbf{s} \times \mathbf{r})^{\top} \mathbf{D}^{-1}(\boldsymbol{\omega} \times \boldsymbol{\Theta} \boldsymbol{\omega}+a \mathbf{s} \times \mathbf{r}-\boldsymbol{\Phi}(t)(\mathbf{s}-\mathbf{r}))
\end{aligned}
$$

Taking into account negative definiteness of the matrix $\mathrm{D}$, we arrive at the estimate

$$
\begin{aligned}
& \dot{V}_{1} \leq-\left(\beta_{1} \gamma h-\frac{\beta_{2}}{h}\right)\|\boldsymbol{\omega}\|^{2}-\frac{\beta_{3}}{h}\|\mathbf{s} \times \mathbf{r}\|^{2} \\
& +\frac{\beta_{4}}{h}\|\mathbf{s}-\mathbf{r}\|\|\boldsymbol{\omega}\|^{2}+\beta_{5} \gamma\|\mathbf{s}-\mathbf{r}\|\|\boldsymbol{\omega}\| \\
& -\frac{1}{h}(\mathbf{s} \times \mathbf{r})^{\top} \mathbf{D}^{-1} \mathbf{\Phi}(t)(\mathbf{s}-\mathbf{r}),
\end{aligned}
$$

where $\beta_{1}, \beta_{2}, \beta_{3}, \beta_{4}, \beta_{5}$ are positive constants.

There exists a number $\delta>0$ such that

$$
\begin{aligned}
& \dot{V}_{1} \leq-\left(\beta_{1} \gamma h-\frac{2 \beta_{2}}{h}\right)\|\boldsymbol{\omega}\|^{2}-\frac{\beta_{3}}{2 h}\|\mathbf{s}-\mathbf{r}\|^{2} \\
& +\beta_{5} \gamma\|\mathbf{s}-\mathbf{r}\|\|\boldsymbol{\omega}\|-\frac{1}{h}(\mathbf{s} \times \mathbf{r})^{\top} \mathbf{D}^{-1} \mathbf{\Phi}(t)(\mathbf{s}-\mathbf{r})
\end{aligned}
$$

for $\|\mathbf{s}-\mathbf{r}\|<\delta$.

Next, applying the approach developed in [Aleksandrov, 1996; Tikhomirov, 2007], define a Lyapunov function by the formula

$$
V_{2}(t, \boldsymbol{\omega}, \mathbf{s})=V_{1}(\boldsymbol{\omega}, \mathbf{s})+\frac{1}{h}(\mathbf{s} \times \mathbf{r})^{\top} \mathbf{D}^{-1} \mathbf{J}(t, \varepsilon)(\mathbf{s}-\mathbf{r}) .
$$

Here $\varepsilon$ is a positive parameter, and

$$
\mathbf{J}(t, \varepsilon)=\int_{0}^{t} \exp (-\varepsilon(t-\tau)) \boldsymbol{\Phi}(\tau) d \tau .
$$

Consider the derivative of $V_{2}(t, \boldsymbol{\omega}, \mathbf{s})$ with respect to system (2), (6). We have

$$
\begin{aligned}
& \dot{V}_{2} \leq-\left(\beta_{1} \gamma h-\frac{2 \beta_{2}}{h}\right)\|\boldsymbol{\omega}\|^{2}-\frac{\beta_{3}}{2 h}\|\mathbf{s}-\mathbf{r}\|^{2} \\
& +\left(\beta_{5} \gamma+\frac{\beta_{6}}{h \varepsilon}\right)\|\mathbf{s}-\mathbf{r}\|\|\boldsymbol{\omega}\|+\frac{\beta_{7} \varepsilon}{h}\|\mathbf{J}(t, \varepsilon)\|\|\mathbf{s}-\mathbf{r}\|^{2}
\end{aligned}
$$

for $\|\mathbf{s}-\mathbf{r}\|<\delta$, where $\beta_{6}$ and $\beta_{7}$ are positive constants. It is known, see [Bogoliubov and Mitropolsky, 1961], that $\varepsilon\|\mathbf{J}(t, \varepsilon)\| \rightarrow 0$ as $\varepsilon \rightarrow 0$ uniformly with respect to $t \geq 0$. Therefore, we can choose and fix sufficiently small values of $\gamma$ and $\varepsilon$ and after that find a sufficiently large number $h_{0}>0$ such that

$$
\begin{aligned}
& \frac{\gamma \eta_{1}}{4}\|\boldsymbol{\omega}\|^{2}+\frac{1}{4}\|\mathbf{s}-\mathbf{r}\|^{2} \leq V_{2} \leq \gamma \eta_{2}\|\boldsymbol{\omega}\|^{2}+\|\mathbf{s}-\mathbf{r}\|^{2}, \\
& \dot{V}_{2} \leq-\frac{1}{2} \beta_{1} \gamma h\|\boldsymbol{\omega}\|^{2}-\frac{\beta_{3}}{4 h}\|\mathbf{s}-\mathbf{r}\|^{2}
\end{aligned}
$$

for $h \geq h_{0},\|\mathbf{s}-\mathbf{r}\|<\delta$. Here

$$
\eta_{1}=\min \{A, B, C\}, \quad \eta_{2}=\max \{A, B, C\} .
$$

Thus, function $V_{2}(t, \boldsymbol{\omega}, \mathbf{s})$ satisfies all the conditions of the Lyapunov asymptotic stability theorem. This completes the proof.

Remark 1. Theorem 1 states that the destabilizing effect of a nonstationary perturbation in restoring torque can be compensated via choosing a sufficiently large multiplier at the vector of dissipative torque.

\section{Nonlinear Restoring and Perturbing Torques}

Next, consider the case where parameter $\mu$ in equations (5) is greater than one. Hence, restoring and perturbing torques are essentially nonlinear.

It is worth mentioning that mechanical systems with essentially nonlinear control laws were studied, for example, in [Rivin, 2003; Luongo, 2015]. It is known [Aleksandrov, Aleksandrova and Zhabko, 2013; Peuteman and Aeyels, 2011] that such control laws are more robust with respect to impact of delay and nonstationary perturbations than linear ones.

Theorem 2. Let Assumption 1 be fulfilled. If $\mu>1$, then the equilibrium position (3) of system (2), (5) is asymptotically stable.

Proof. Choose a Lyapunov function candidate in the form (7), where $\gamma$ is a positive parameter. The deriva- 
tive of this function with respect to system (2), (5) is

$$
\begin{aligned}
& \dot{V}_{1}=\gamma h \boldsymbol{\omega}^{\top} \mathbf{D} \boldsymbol{\omega}-\gamma a\|\mathbf{s}-\mathbf{r}\|^{\mu-1} \boldsymbol{\omega}^{\top}(\mathbf{s} \times \mathbf{r}) \\
& +\gamma \boldsymbol{\omega}^{\top} \mathbf{\Phi}(t) \mathbf{Q}(\mathbf{s}-\mathbf{r})+\frac{1}{h}((\boldsymbol{\omega} \times \mathbf{s}) \times \mathbf{r})^{\top} \mathbf{D}^{-1} \boldsymbol{\Theta} \boldsymbol{\omega} \\
& +\frac{1}{h}(\mathbf{s} \times \mathbf{r})^{\top} \mathbf{D}^{-1}(\boldsymbol{\omega} \times \boldsymbol{\Theta} \boldsymbol{\omega}-\mathbf{\Phi}(t) \mathbf{Q}(\mathbf{s}-\mathbf{r})) \\
& +\frac{1}{h} a\|\mathbf{s}-\mathbf{r}\|^{\mu-1}(\mathbf{s} \times \mathbf{r})^{\top} \mathbf{D}^{-1}(\mathbf{s} \times \mathbf{r})
\end{aligned}
$$

If the value of $\gamma$ is sufficiently large, then there exist positive numbers $\delta, \beta_{1}, \beta_{2}$ such that

$$
\begin{aligned}
& \dot{V}_{1} \leq-\beta_{1} \gamma\|\boldsymbol{\omega}\|^{2}-\beta_{2}\|\mathbf{s}-\mathbf{r}\|^{\mu+1} \\
& -\frac{1}{h}(\mathbf{s} \times \mathbf{r})^{\top} \mathbf{D}^{-1} \mathbf{\Phi}(t) \mathbf{Q}(\mathbf{s}-\mathbf{r})
\end{aligned}
$$

for $\|\mathbf{s}-\mathbf{r}\|<\delta$.

Next, construct a Lyapunov function in the form

$V_{2}(t, \boldsymbol{\omega}, \mathbf{s})=V_{1}(\boldsymbol{\omega}, \mathbf{s})+\frac{1}{h}(\mathbf{s} \times \mathbf{r})^{\top} \mathbf{D}^{-1} \mathbf{J}(t, \varepsilon) \mathbf{Q}(\mathbf{s}-\mathbf{r})$,

where $\varepsilon$ is a positive parameter, and the matrix $\mathbf{J}(t, \varepsilon)$ is defined by formula (8). We obtain

$$
\begin{aligned}
& \dot{V}_{2} \leq-\beta_{1} \gamma\|\boldsymbol{\omega}\|^{2}-\beta_{2}\|\mathbf{s}-\mathbf{r}\|^{\mu+1} \\
& +\frac{\beta_{3}}{\varepsilon}\|\mathbf{s}-\mathbf{r}\|^{\mu}\|\boldsymbol{\omega}\|+\beta_{4} \varepsilon\|\mathbf{J}(t, \varepsilon)\|\|\mathbf{s}-\mathbf{r}\|^{\mu+1}
\end{aligned}
$$

for $\|\mathbf{s}-\mathbf{r}\|<\delta$, where $\beta_{3}>0, \beta_{4}>0$.

Hence, one can choose a sufficiently large value of $\gamma$ and sufficiently small values of $\delta$ and $\varepsilon$ such that

$$
\begin{aligned}
& \frac{\gamma \eta_{1}}{4}\|\boldsymbol{\omega}\|^{2}+\frac{1}{4}\|\mathbf{s}-\mathbf{r}\|^{2} \leq V_{2} \leq \gamma \eta_{2}\|\boldsymbol{\omega}\|^{2}+\|\mathbf{s}-\mathbf{r}\|^{2} \\
& \dot{V}_{2} \leq-\frac{1}{2} \beta_{1} \gamma\|\boldsymbol{\omega}\|^{2}-\frac{\beta_{2}}{2}\|\mathbf{s}-\mathbf{r}\|^{\mu+1}
\end{aligned}
$$

for $\|\mathbf{s}-\mathbf{r}\|<\delta$. Here constants $\eta_{1}$ and $\eta_{2}$ are defined by formulae (9). This completes the proof.

Remark 2. In contrast to Theorem 1, in Theorem 2 it is not required that parameter $h$ be sufficiently large. In the case where $\mu>1$, asymptotic stability of the equilibrium position (3) of system (2), (5) is guaranteed for an arbitrary $h>0$.

\section{Simulation Results}

In this section, we demonstrate the previous theoretical results by means of a numerical simulation. Let the inertial parameters of a rigid body are given as: $A=5$,
$B=6, C=4$. The programmed orientation (3) of the body is such that direction cosines $\gamma_{1}, \gamma_{2}, \gamma_{3}$ of the axis $z$ in the inertial coordinate system are equal to each other and equal to $1 / \sqrt{3}$.

Consider the control process governed by system (2), (6). Choose the matrix $\mathbf{D}$ of dissipative torque in the form $\mathbf{D}=-\operatorname{diag}\{1,1,1\}$. Let $a=1$, and the disturbing torque be taken in the form $\widetilde{\mathbf{M}}=b \sin \left(\omega_{0} t\right) \mathbf{s} \times \mathbf{r}$. We assume that $b=2$ ( $b$ is two times greater than $a$ ), and $\omega_{0}$ is rather small: $\omega_{0}=0.3$. The initial values of "aircraft" angles $\varphi(0)=0.5, \psi(0)=0.6$, $\theta(0)=0.8$ result in the following initial values of direction cosines: $\gamma_{1}(0)=0.717356, \gamma_{2}(0)=0.334019$, $\gamma_{3}(0)=0.611418$. The initial values of angular velocity projections are $\omega_{x}(0)=\omega_{y}(0)=\omega_{z}(0)=1$.

Choosing the value of parameter $h$, we first take the value $h=2$. The results of numerical integration (Figs. $1,2)$ show that control process does not converge to the programmed orientation (3).

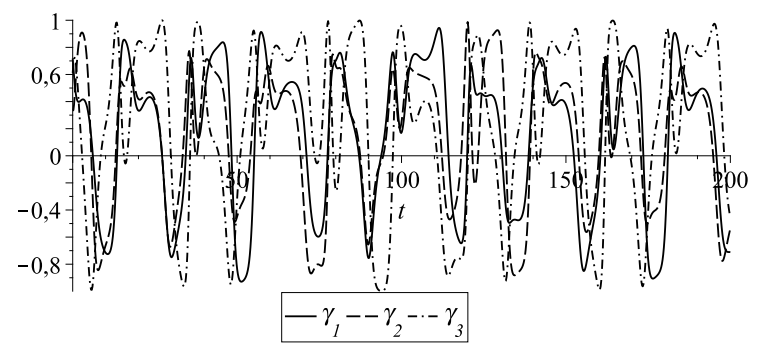

Figure 1. Direction cosines time history, $h=2$

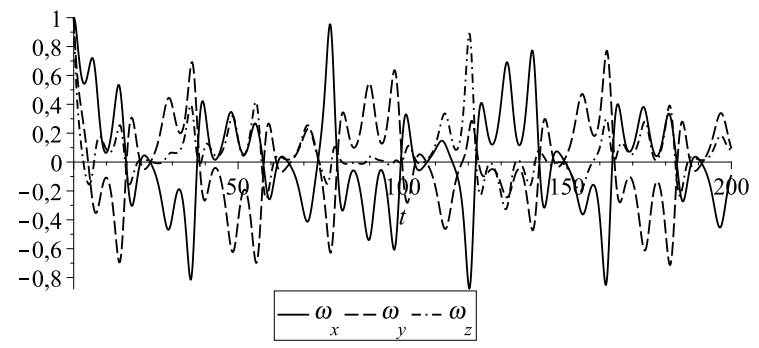

Figure 2. Angular velocity time history, $h=2$

Then we choose $h=3$ and repeat calculations with the same initial conditions. In accordance with Theorem 1 and Remark 1, Figs. 3, 4 demonstrate that the equilibrium position (3) is asymptotically stable.

\section{Conclusion}

In the present paper, the problem of monoaxial attitude stabilization of a rigid body subjected to time- 


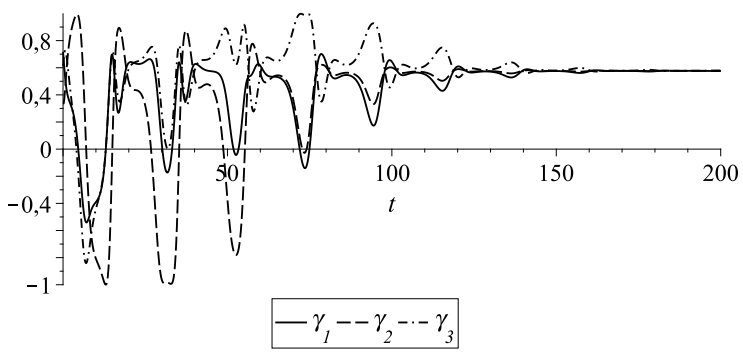

Figure 3. Direction cosines time history, $h=3$

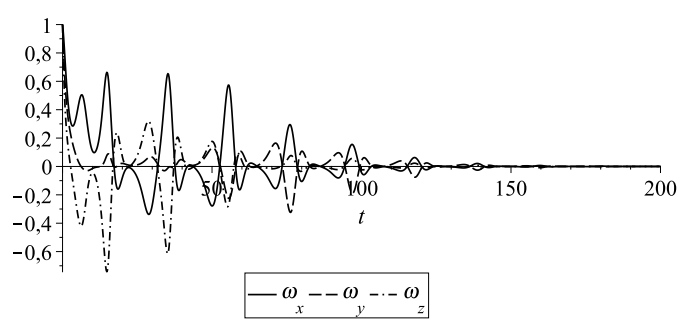

Figure 4. Angular velocity time history, $h=3$

varying perturbations was studied. The considered perturbations are represented as linear combinations of homogeneous functions with time-varying coefficients. It is assumed that the orders of perturbations coincide with those of components of restoring torque, and coefficients possess zero mean values. In particular, coefficients of a such type may describe periodic or almost periodic oscillations. It is worth mentioning that the stability conditions derived in this paper do not impose any restrictions on amplitudes of these oscillations.

It is worth mentioning that a magneto-Coulombic attitude control system for a satellite stabilization was exploited in [Giri and Sinha, 2017]. The averaging method was used for the stability analysis of a programmed motion. Meanwhile, for justification the possibility of such an approach, the authors artificially introduce a small parameter at control torque. This results in the necessity of an additional assumptions, such as isoinertial mass distribution and the absence of disturbing torques. We believe that our approach to the application of the averaging method is more effective, and it can be used for the problem considered in [Giri and Sinha, 2017] without limitations mentioned above.

\section{Acknowledgements}

The reported study was financially supported by Government of Russian Federation (Grant No. 08-08) and by the Russian Foundation for Basic Research (Grant No. 17-01-00672-a).

\section{References}

Akulenko, L.D., Leshchenko, D.D., and Chernous'ko, F.L. (1986) Perturbed motions of a rigid body that a close to regular precession. Mech. of solids, 21(5), pp. $1-8$.

Aleksandrov, A.Yu. (1996) On the stability of equilibrium of unsteady systems. Prikladnaya Matematika $i$ Mekhanika, 13(2), pp. 205-209. (in Russian).

Aleksandrov, A.Y. (2001) Stability of complex systems in critical cases. Automation and Remote Control, 62(9), pp. 1397-1406.

Aleksandrov, A.Y., Aleksandrova, E.B., and Zhabko, A.P. (2013) Stability analysis for a class of nonlinear nonstationary systems via averaging. Nonlinear Dyn. Syst. Theory, 13(4), pp. 332-343.

Aleksandrov, A.Yu., Kosov, A.A., and Chen, Y. (2011) Stability and stabilization of mechanical systems with switching. Automation and Remote Control, 72(6), pp. 1143-1154.

Antipov, K.A., and Tikhonov, A.A. (2014) Electrodynamic control for spacecraft attitude stability in the geomagnetic field. Cosmic Research, 52 (6), pp. 472 480.

Antonchik, V.S. (1983) On the problem of control of a gyroscopic stabilization system. In Mathematical Methods of Optimization and Control in Complex Systems. Kalinin State University, Kalinin, pp. 8690. (in Russian).

Bogoliubov, N.N., and Mitropolsky, Y.A. (1961) Asymptotic Methods in the Theory of Non-linear Oscillations. Gordon and Breach. New York.

Britton, N.F. (2003) Essential Mathematical Biology. Springer-Verlag. London.

Chikhachev, A. (2017) The non-stationary selfconsistent model of the charged system. Cybernetics and Physics, 6(2), pp. 64-70.

Giri, D.K., and Sinha, M. (2017) Three-axis attitude control of Earth-pointing isoinertial magnetoCoulombic satellites. Int. J. Dynam. Control, 5, pp. 644-652.

Grebennikov, E.A. (1986) The Averaging Method in Applied Problems. Nauka. Moscow. (in Russian).

Guckenheimer, J., and Holmes, P. (1983) Nonlinear Oscillations, Dynamical Systems, and Bifurcations of Vector Fields. Springer-Verlag, Berlin, Heidelberg, New York, Tokyo.

Khapaev, M.M. (1993) Averaging in Stability Theory. Kluwer. Dordrecht.

Kosjakov, E.A., and Tikhonov, A.A. (2015) Differential equations for librational motion of gravityoriented rigid body. Int. J. Non-Linear Mech., 73(1), pp. 51-57.

Kosov, A.A. (2007) The exponential stability and stabilization of non-autonomous mechanical systems with non-conservative forces. J. Appl. Math. Mech. 71(3), pp. 371-384.

Kovaleva, A. (2016) Autoresonance in Klein-Gordon 
chains. Cybernetics and Physics, 5(4), pp. 123-129.

Krasilnikov, P.S., and Amelin, R.N. (2016) On Saturn's rotation relative to a center of mass under the action of the gravitational moments of the Sun and Jupiter. Cosmic Research. 54(2), pp. 127-133.

Luongo, A., and Zulli, D. (2015) Nonlinear energy sink to control elastic strings: The internal resonance case. Nonlinear Dynamics, 81(1-2), pp. 425-435.

Manevich, L., Smirnov, V., and Romeo, F. (2016) Non-stationary resonance dynamics of the harmonically forced pendulum. Cybernetics and Physics, 5(3), pp. 91-95.

Markeyev, A.P. (2011) The equations of the approximate theory of the motion of a rigid body with a vibrating suspension point. J. Appl. Math. Mech., 75(2), pp. 132-139.

Ma-yu-shan, V., and Altsybeyev, V. (2016) On improving the particle-in-cell simulations accuracy for sources of charged particles. Cybernetics and Physics, 5(1), pp. 16-20.

Nayfeh, A.H. (1981) Introduction to Perturbation Techniques. Wiley-Interscience. New York.

Ovchinnikov, M.Yu., Roldugin, D.S., and Penkov, V.I. (2012) Asymptotic study of a complete magnetic attitude control cycle providing a single-axis orientation Acta Astronautica, 77, pp. 48-60.

Peuteman, J., and Aeyels, D. (1999) Averaging results and the study of uniform asymptotic stability of homogeneous differential equations that are not fast time-varying. SIAM J. Control Optim., 37(4), pp. 997-1010.

Peuteman, J., and Aeyels, D. (2011) Averaging tech- niques without requiring a fast time-varying differential equation. Automatica, 47, pp. 192-200.

Rivin, E.I. (2003) Passive Vibration Isolation. ASME Press. New York.

Schaub, H., and Junkins, J.L. (2009) Analytical Mechanics of Space Systems. American Institute of Aeronautics and Astronautics. Reston. Virginia.

Tikhomirov, O.G. (2007) Stability of homogeneous nonstationary odinary differential equations systems. Vestnik St. Petersburg Univ. Ser. 10, (3), pp. 123-130. (in Russian).

Tikhonov, A.A. (2000) Resonance phenomena in oscillations of a gravity-oriented rigid body. Part 4: Multifrequency resonances. Vestnik Sankt-Peterburgskogo Universiteta. Ser 1. Matematika Mekhanika Astronomiya, (1), pp. 131-137.

Tikhonov, A.A. (2002) Refinement of the oblique dipole model in the evolution of rotary motion of a charged body in the geomagnetic field. Cosmic Research, 40 (2), pp. 157-162.

Tikhonov, A.A. (2004) On the rotary motion of a shielded artificial earth satellite in a noncentral gravitational field. Vestnik Sankt-Peterburgskogo Universiteta. Ser 1. Matematika Mekhanika Astronomiya, (3), pp. 81-87.

Tikhonov, A.A. (2005) Secular evolution of rotary motion of a charged satellite in a decaying orbit. Cosmic Research, 43(2), pp. 107-121.

Zubov, V.I. (1978) Theorie de la Commande. Mir. Moscow. (in French).

Zubov, V.I. (1999) Theory of Oscillations. World Scientific. Singapore etc. 